\section{$\underset{\substack{\text { hommes } \\ \text { \& migrations }}}{ }$}

\section{Hommes \& migrations}

Revue française de référence sur les dynamiques

migratoires

1305 | 2014

L'exil chilien en France

\title{
Hommage à Ibrahima Sylla
}

\section{François Bensignor}

\section{CpenEdition \\ Journals}

\section{Édition électronique}

URL : http://journals.openedition.org/hommesmigrations/2769

DOI : 10.4000/hommesmigrations.2769

ISSN : 2262-3353

\section{Éditeur}

Musée national de l'histoire de l'immigration

\section{Édition imprimée}

Date de publication : 1 janvier 2014

Pagination : 186-192

ISBN : 978-2919-040261

ISSN : $1142-852 X$

\section{Référence électronique}

François Bensignor, «Hommage à Ibrahima Sylla », Hommes \& migrations [En ligne], 1305 | 2014, mis en ligne le 11 juillet 2014, consulté le 22 septembre 2020. URL : http://journals.openedition.org/ hommesmigrations/2769; DOI : https://doi.org/10.4000/hommesmigrations.2769 


\title{
HOMMAGE À IBRAHIMA SYLLA
}

\author{
FRANÇOIS BENSIGNOR
}

L a disparition du fondateur du label Syllart Productions, survenue le 30 décembre 2013, est une perte pour le milieu des musiques du monde. Ce producteur infatigable a su faire apprécier la musique africaine aux publics occidentaux. On lui doit un trésor de créations, comme l'album Soro de Salif Keïta ou le lancement d'Ismaël Lô, la mise en œuvre des groupes concepts Africando et Mandé Kalou, ou la publication d'archives patrimoniales. En guise d'hommage, nous publions des extraits de l'entretien accordé pour les vingt ans de son label.

Découvreur de talents au flair incomparable, capable de risquer son argent pour faire éclore les plus belles musiques d'Afrique, Ibrahima Sylla était un personnage hors du commun. II menait ses affaires avec poigne, se faisant respecter par ses partenaires comme par ses concurrents. Ses relations avec les artistes étaient particulières, parfois houleuses. Il savait ce qu'il voulait. Ses productions ont porté à la connaissance du monde certaines des facettes les plus somptueuses de la création musicale africaine durant trois décennies. En 2010, alors qu'il se savait déjà malade et condamné, le coffret de 18 CD élaboré par ses soins pour le cinquantenaire des indépendances africaines, Africa, 50 Years of Music, 1960-2010 (Syllart Productions/ Discograph), faisait figure de legs aux générations futures. Huit ans auparavant, alors qu'il célébrait les vingt ans de Syllart Productions, Ibrahima Sylla publiait un coffret de cinq CD rassemblant les perles de son catalogue, sélectionnées avec cette passion pour la musique qui le caractérisait. Sylla avait pour habitude de garder ses distances vis-à-vis des médias. Mais pour cette occasion, il a accepté de maccorder un long entretien. En guise d'hommage à cette personnalité pour laquelle j'ai toujours éprouvé beaucoup de respect malgré les nombreuses critiques dont il était l'objet, j'ai souhaité en reproduire dans ces pages de larges extraits. Ibrahima Sylla s'y montrait généreux en paroles, dévoilant de nombreux éléments de son histoire et de sa personnalité, sur lesquels il avait jusqu'alors observé un silence pudique. À travers ses paroles, on mesure l'attachement passionné qui liait cet l'homme à la musique et aux musiciens.

\section{Le fils d'un grand chef religieux}

"Je suis d'origine diakanké, une branche des Mandingues. Ma mère est une Bambara de Koulikoro [Mali]. Je parle mandingue, ainsi que dioula et bambara, des langues dérivées du mandingue. Mon arrière-grand-père qui venait du Mali s'est installé en Guinée, où est né mon père. Moi, je suis né en Côte d'Ivoire, que j'ai quittée à l'âge de trois ans, et j'ai grandi au Sénégal. Je parle wolof mieux que mandingue. Je parle également très bien le peul. En effet, mon père, Al Hassan Sylla, et son jumeau, Al Houceyne Sylla, avaient chacun quatre épouses, des Peules pour la plupart. Dans cette grande famille, nous sommes 63 enfants. "Nobles et grands chefs religieux, mon père et son frère 


\section{Une relation particulière avec les griots}

"Mon père était tellement riche et tellement généreux à l'égard des griots que tous lui faisaient la cour, Maliens, Guinéens, Sénégalais. Auprès de lui dans ma jeunesse, j'ai connu les griots les plus célèbres, comme Kouyaté Sory Kandia, le Guinéen, ou Fanta Damba, la Malienne. Dans les années 1960, mon père offrait des villas à des griots. En 1967, Fanta

Damba a reçu de lui une Mercedes. Plus tard,

jumeau, à l'origine proches de la confrérie musulmane tidjaniya, pratiquaient un islam tolérant et étaient très liés au grand chef mouride Serigne Fallou Mbacké (second calife des Mourides et fils de Cheikh Amadou Bamba, le fondateur du mouridisme). En 1967, ce dernier convia mon père au Grand Magal de Touba comme invité d'honneur. De toute l'Afrique de l'Ouest, on venait vénérer mon père et son jumeau, que les chefs d'État africains venaient consulter avant de prendre d'importantes décisions.

"Alors que j'étais en $4^{\mathrm{e}}$, mon père a décidé de me retirer de l'école pour m'emmener au Tchad, où j'ai passé un an à approfondir mon éducation coranique. J'étais alors un jeune Dakarois privilégié, imbu de sa personne, qui se faisait conduire à l'école en Mercedes. Mon père a voulu casser mon orgueil et me ramener à la réalité. II m’a inculqué I'humilité, le partage. II m'a fait voyager avec lui au Cameroun, au Zaïre, en Centrafrique, au Togo, au Bénin. Au Sénégal, je ne connaissais que les musiques afro-cubaines et la soul américaine, et je prenais pour des sauvages ceux qui n'écoutaient pas ces musiques-là. En voyageant auprès de mon père pendant quatre ans, j'ai compris qu'il y avait autre chose, j'ai découvert d'autres cultures. C'est après cette période, en 1974, que mon père m’a envoyé en France, où j'ai étudié l'économie et la gestion. dans mon métier de producteur, il a fallu que je cache cette réalité aux griots musiciens que j'ai rencontrés. Si je leur avais dit qui était mon père, nos relations auraient changé. II n'aurait plus été question de cachets d'artiste, mais bien de leur donner à profusion. "Lorsque j’ai produit l'album des femmes africaines, par exemple, j'ai approché Mahawa Kouyaté - la femme de Soudioulou Sissokho, l'un des meilleurs joueurs de cora dans les années 1950, premier artiste africain à devenir membre de la Sacem -, qui était l'une des griotes de mon père. Elle n'a jamais voulu accepter aucun contrat de ma part. J'ai donc préféré m'abstenir de l'enregistrer. Plus tard, à Bamako, j'ai rencontré la vieille Fanta Damba, à qui je voulais produire un disque. Elle m'a rappelé qui était mon père et j'ai préféré en rester là. Si un griot chante pour moi, toute ma vie je lui devrais moralement quelque chose. Je deviendrais son obligé." Ibrahima Sylla s'est toutefois toujours intéressé à produire des griots. II a notamment publié d'excellents albums de Kandia Kouyaté et de Kasse Mady Diabaté, qui les ont fait connaître à l'international. En 2004, il lance le concept Mandekalou, réunissant dans un lieu à la campagne proche de Bamako certains des griots les plus en vue de l'époque, afin qu'ils gravent pour la postérité de grandes œuvres du patrimoine mandingue. II en résultera deux superbes volumes parus successivement en 2004 et en 2006. 


\section{MUSIQUES}

\section{Une grande passion pour la musique}

"Étudiant en France, je collectionnais les disques de musique cubaine. Nous nous retrouvions avec une bande de passionnés chez Pasdeloup, célèbre magasin de disques près du Luxembourg, autour de Chérif Salif Sy, l'un des vendeurs [devenu conseiller du président sénégalais Abdoulaye Wade à l'époque de l'entretien]. Ce sont eux qui m'ont poussé à concevoir le projet d'Africando. À partir de mes collections, un autre passionné a commencé à faire des compilations et c'est ainsi que j'ai pris goût à la fabrication de disques.

“À mon retour au Sénégal en 1979, j'étais décidé à faire de la production musicale. II fallait que j'en informe mon père. Mais chez nous, lorsqu'il s'agit de grandes décisions de la vie, il faut passer par les griots. J'ai donc expliqué au griot de mon père, Mamadou Kouyaté, grand maître de la kora et père de Soryba Kouyaté, ce que je voulais faire, afin qu'il le dise à mon père et que celui-ci m’aide à démarrer. Le vieux griot est allé parler à mon père, mais ses mots ont été mal interprétés. Croyant que je voulais me mettre à la chanson et ne m'ayant pas sous sa main, mon père a giflé son griot. Aussitôt, j'ai quitté la maison et, pendant trois ou quatre ans, nous ne nous sommes plus adressé la parole, mon père et moi. Dans cette épreuve, ma sœur Bintou Sylla m'a toujours été un précieux soutien, et plus tard mon père a reconnu mon statut de businessman.

“J'ai commencé à travailler au studio Golden Baobab de Francis Arphan Senghor [fils du président Léopold Sédar Senghor], où j'ai fait les albums du groupe l'Étoile de Dakar avec Youssou N'Dour à ses débuts, de l'Orchestre Baobab, d'Ouza et de Guelewar pour les productions Jambaar (les

"valeureux", les "guerriers") fondées avec Ibrahima Fall. En commençant ce métier, je me suis dit : 'Mon père a tout donné aux griots, moi je vais travailler avec eux.' Mais à la différence de mon père qui aimait qu'on chante ses louanges, j'ai toujours demandé aux artistes de ne pas citer mon nom dans leurs disques. Si je l'entends au mixage, je l'enlève.

\section{Produire les artistes pour rassembler les Africains}

“En 1981, j’ai décidé de créer mon propre label, Syllart Productions, sur lequel j'ai produit les nouveaux albums de l'Orchestre Baobab et d'Ismaël Lô. En 1983, je m'installe à Paris, où j'ouvre un magasin de disques, Kubaney Musique, rue de Rocroy dans le $X^{e}$ arrondissement. C'est là que je commence à produire mes premiers Zaïrois, Bopol Mansiamina, Empire Bakuba, Quatre Étoiles, Nyboma, mais aussi les Cap-Verdiens de Cabo Verde Show, le salsero béninois Gnonnas Pedro, l'Ivoirienne Nyanka Bell, ainsi que Rasta Poué, le premier album d'Alpha Blondy. “Pour mes productions, j'ai deux grandes règles : ne signer de contrat avec un artiste que pour un seul album ; 
faire évoluer la musique, changer les habitudes, ne pas faire ce que tout le monde fait. Avant l'enregistrement d'un album, je veux en connaître la conception artistique. Pendant les prises, je laisse faire. Je viens à la fin et je suis toujours là pour le mixage de mes albums. Les musiciens apprécient généralement de me voir m'impliquer. S'ils ne me voient pas, ils ont peur, surtout les arrangeurs.

"Je ne suis pas musicien. Je ne lis pas la musique, je ne l'écris pas non plus et je ne joue d'aucun instrument, mais je me dis que j'ai peut-être un don. Quel que soit le musicien ou la musique, je comprends et j'ai toujours une petite idée à donner qui fait la différence. Certains pensent que c'est grâce aux gris-gris de mon père, je crois plutôt que c'est à force d'écouter beaucoup de musique. Quand j'ai fini l'enregistrement et le mixage, ma grande satisfaction, c'est d'être le premier et le seul à pouvoir écouter l'album. J'ai investi de mon argent et de mon temps et c'est comme si je m'installais dans la maison que je viens de bâtir. Le son est bien. Tout est net, tout est clair : c'est la musique, c'est un voyage... "L'Afrique est un vaste continent avec de multiples pays, des milliers d'ethnies, de races et de langues. À l'échelle africaine, j'ai essayé d'unir et de rassembler les Africains autour de la musique. C'est ma façon de contribuer à l'organisation de l'unité africaine."

Ibrahima Sylla évoque des artistes qu'il a produits et accompagnés :

\section{/// Ismaël Lô (Sénégal)}

“Ismaël Lô est pour moi comme un frère. À mes débuts, en 1981, j'ai produit son premier album L'Homme orchestre, Gor Sayina. Nous étions très jeunes. Peu après, il est parti travailler comme guitariste dans un hôtel en Espagne. À son retour au Sénégal, j'étais déjà installé en France et il a intégré le Super Diamono, pour le quitter dans des conditions assez rocambolesques. À l'époque on a dit que j'avais cassé le groupe. Aujourd'hui, je voudrais remettre les pendules à l'heure. J'étais venu à Dakar pour produire le Super Diamono. Mais les responsables du groupe mont dit qu'ils avaient déjà un contrat avec un producteur pour enregistrer en France. J'allais abandonner quand Ismaël m’a proposé de le produire."

Une relation de fidélité et d'amitié lit les deux hommes et Ibrahima Sylla a produit ses premiers albums : L'Homme orchestre, Gor Sayina (1981), Xalatt (1983), Xiif (1985), Natt (1987) et Diawar (1989). “Et puis un jour, j’ai dit à Ismaël : 'Nous avons fait cinq albums ensemble. Il faut aller plus loin. Je pense qu'il te faut signer avec une major.' C'est moi qui l'ai emmené chez Barclay voir Philippe Constantin, avec qui il a signé. J’ai refusé la direction artistique du premier album qui m'était proposée, mais j'ai accepté celle du second, Dibi Dibi Rek."

\section{/// Alpha Blondy (Côte d'Ivoire)}

"C'était au début de ma carrière, en 1983-1984. Je voulais sortir en France le premier album d'Alpha Blondy, Brigadier Sabari, qui s'était très bien vendu à Abidjan. Son producteur avait une importante dette envers la société ivoirienne des droits d'auteur affiliée à la Sacem. II m’a proposé les droits d'exploitation du disque en France contre le remboursement de cette dette. J'ai fait le nécessaire auprès de la Sacem, j'ai récupéré 45000 pochettes du disque à l'usine EMI d'Abidjan et j'ai tout vendu. Alpha Blondy est venu me voir dans mon magasin de disques à Paris. Je lui ai proposé un contrat et il m'a proposé d'acheter Rasta Poué, le single qu'il venait d'enregistrer. Je l'ai inclus dans l'album Brigadier Sabari, contre 40000 francs qu'il a utilisés pour aller en Jamaïque enregistrer Cocody Rock, son premier album signé chez EMI."

\section{/// Pépé Kalle}

\section{(République démocratique du Congo}

“J'ai connu Pépé Kalle en 1985 grâce à Nyboma, que je produisais déjà dans Quatre Étoiles et qui avait fait un album solo avec moi. Nyboma voulait 


\section{MUSIQUES}

que je fasse un album avec lui et Pépé Kalle. J'hésitais, parce que Pépé Kalle était dans l'orchestre Empire Bakuba. Mais Nyboma m'a rappelé que son association avec Pépé Kalle remontait aux années 1970 avec l'orchestre Lipua Lipua de Kinshasa. Là-dessus, mon grand ami Bopol Mansiamina a achevé de me convaincre. J'ai profité du passage de l'Empire Bakuba à Paris pour aller voir Pépé Kalle et nous avons décidé de faire l'album Zouke Zouke. C'était le début d'une longue collaboration et d'une grande amitié. Pépé Kalle était un type très généreux et très chahuteur. Nous sommes devenus intimes et nous avions des relations quasi fraternelles. Quand j'ai appris sa mort, j'ai eu un grand choc, parce que c'était quelqu'un que j'aimais vraiment beaucoup."

\section{/// Salif Keïta (Mali)}

"Salif Keïta est le premier artiste malien que j'ai produit. Depuis 1983, je voulais faire un album avec lui dans le style des Ambassadeurs, mais ça ne marchait pas. Salif tergiversait entre les propositions que lui faisaient les éditions Virgin. En 1986, Mamadou Konté [le fondateur d'Africa Fête], devenu manager de Salif, mannonce qu'il est prêt si je suis toujours intéressé à le produire. Je vois Salif qui me dit :'Je ne te demande pas d'argent. Je veux un bon produit. II me faut un budget de 250000 francs, un grand arrangeur, un studio à plein temps où je travaille comme je veux.' II avait déjà fait des titres avec JeanPhilippe Rykiel, et Béatrice Soulé nous a présenté François Bréant [musicien arrangeur, qui avait travaillé avec Bernard Lavilliers]. Tous deux ont beaucoup apporté dans cet album. "Nous étions tous d'accord, mais selon moi, la production ne devait même pas atteindre les 250000 francs. J'ai donc refusé d'inscrire la somme sur le contrat, comme le voulait Salif. On commence l'album sans contrat. On laisse venir les choses en studio et, au bout de deux ou trois mois, je fais mes comptes : on en était à 700000 francs et l'album n'était pas fini... Ce jour-là, j'ai regretté de ne pas avoir inscrit 250000 francs sur le contrat.

"Soro m'a ouvert de nombreuses portes. J'ai commencé à produire intensément au Mali après cet album, qui m'a également apporté une certaine notoriété internationale. II a permis la reconnaissance des musiques africaines par de grands noms comme Carlos Santana, Miles Davis ou Quincy Jones."

\section{/// Kasse Mady Diabaté (Mali)}

" Kasse Mady Diabaté est originaire de Kéla, dans cette partie de la vallée du Niger comprise entre Kangaba et Siguiri à cheval entre le Mali et la Guinée, connue pour être la région des griots orateurs. Issu d'une grande lignée de griots, dont la célèbre Sira Mory Diabaté, il s'est d'abord fait connaître comme l'un des plus grands chanteurs de sa génération au sein de l'Orchestre Badéma

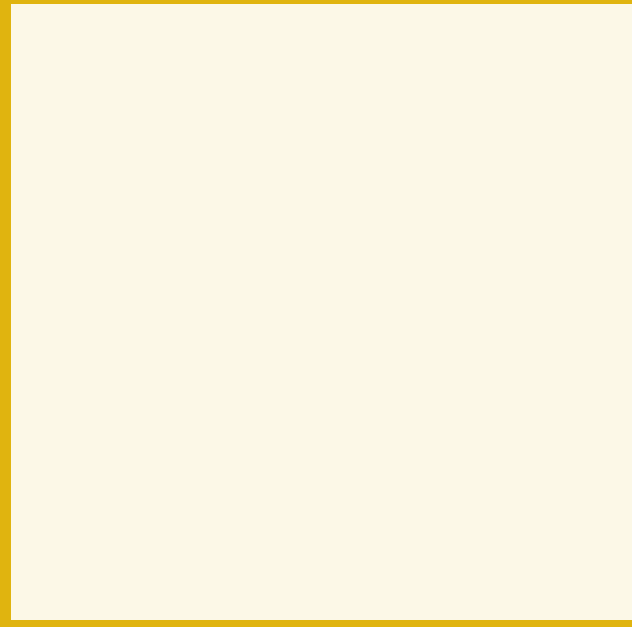

Pepe Kalle (c) D.R 
absolument que je sorte son album parce que d'autres artistes sont en train de reprendre ses chansons. J'ai attendu février pour le sortir. Et de ma vie je n'ai jamais vendu autant de cassettes en si peu de temps. J'ai écoulé plus de 600 ooo cassettes légales. Du coup, j'ai offert une 505 [Peugeot] à Oumou Sangare..."

\section{/// Boubacar Traoré (Mali)}

"La première cassette de Boubacar Traoré a été entièrement produite par Abdoulaye Samassa. Je ne m'étais occupé que du financement. Quand elle est sortie au Mali en 1987, elle n'a pas marché. Quatre ans plus tard,

National. « Lorsque nous avons fait son deuxième album, Kéla, je lui ai demandé de chanter Koulandjan. II me répond: 'Koulandjan de la ville ou le vrai Koulandjan du village?" Je voulais le vrai 'Koulandjan'.'Alors tu en auras pour trente minutes de chant et je ne peux pas le faire sans en demander l'autorisation au village.' II a fallu attendre deux semaines le retour de la lettre du village lui donnant l'autorisation, accompagnée d'une cassette sur laquelle on lui disait les parties à ne pas oublier. Lors de l'enregistrement, je lui ai demandé d'arrêter au bout de treize minutes et trente secondes. II dit alors dans la chanson :'Je m'arrête parce que le propriétaire du studio m'a dit d'arrêter de parler. Je demande pardon aux chasseurs qui m'ont transmis les paroles, mais je continuerai prochainement.' Beaucoup de gens ont chanté Koulandjan, mais personne comme Kasse Mady."

\section{/// Oumou Sangare (Mali)}

"Le premier album d'Oumou Sangare a été enregistré en 1988 à Abidjan sous la direction d'Abdoulaye Samassa [producteur implanté à Bamako], sans que j'aie à m'en occuper. Quant à moi, j'ai manqué de flair. J'ai gardé pendant deux ans la bande. Je ne voulais pas la sortir. Je ne sentais pas cette musique du Wassoulou... Un jour, Oumou Sangare m'appelle en pleurs, me disant qu'il faut
Robert Urbanus, le patron de Sterns [le plus important label de musique africaine du marché anglophone], vient me voir à mon bureau et repart avec une vingtaine de cassettes. Peu après, il m'appelle pour savoir si c'est moi qui ai produit Boubacar Traoré. 'Je ne connais pas !' Il revient me voir, me montre la cassette et me dit qu'il veut en faire un disque. 'Pas de problème!' Quelques mois plus tard, dans son bureau à Londres, je vois les disques de Boubacar Traoré partout sur les étagères, avec une autre pochette. II me dit que ça marche très fort. 'Quoi ?' C'est ce jour-là que j’ai écouté le disque de Boubacar Traoré. Je le lui ai donné en licence." "Robert m'appelle un jour pour me dire que Ry Cooder est intéressé à travailler avec Boubacar Traoré.'Qui est Ry Cooder ?'Il m’explique que c'est un grand guitariste américain [celui qui est à l'origine de Buena Vista Social Club]. Son manager vient alors me rencontrer à Paris. Là, j'ai été trop gourmand. J'ai demandé beaucoup d'argent. II a eu peur. Ry Cooder est allé chercher Ali Farka Touré pour faire l'album Talking Timbuktu. Je suis passé à côté !... J'étais trop africain !..."

\section{/// Rail Band (Mali)}

"Géré par la régie des Chemins de fer du Mali, le Rail Band a été formé en 1970 par Tidiane Koné, griot et saxophoniste, à la demande du chef 
de la gare de Bamako, Demba Diallo. Ce groupe mythique a vu défiler ceux qui ont fait connaître la musique malienne au monde entier : Salif Keïta, Mory Kanté, Djelimady Toukara, Sekou Kouyaté... “J'ai pu racheter les droits d'exploitation des enregistrements historiques que le Rail Band a réalisés d'abord à Bamako au temps de Salif Keïta, puis au Nigeria, quand Mory Kante l'a remplacé au chant, et enfin à Paris."

\section{/// Banzoumana Sissoko (Mali)}

"Ce grand griot malien, aveugle de naissance, est une légende du XXe siècle. Ses pouvoirs surnaturels lui permettaient, dit-on, de faire jouer son n'goni à distance. On ne diffuse sa musique à la radio que dans des circonstances graves. "Je l'ai vu une seule fois : un souvenir inoubliable. C'était à Bamako. J'avais demandé à le rencontrer. II est arrivé au Bureau malien des droits d'auteur, où nous étions plusieurs à l'attendre. À sa descente de voiture, on l'a conduit vers nous et sans qu'on lui dise rien, il est venu vers moi et m'a dit : 'C'est Sylla !' J'étais impressionné qu'il me reconnaisse alors qu'il ne me voyait pas, mais j’ai gardé mon self-control pour répondre à son salut. Quand on lui a expliqué que je venais pour signer le contrat d'exploitation des archives sonores nationales, il a dit :'Pour nous la vie est finie. Vous êtes la relève. Travaillez bien derrière nous! Faites connaître à vos frères, à vos semblables, ce que nous avons fait, afin qu'ils perpétuent cette tradition. Je te donne toute ma bénédiction.' Je l'ai remercié Deux mois après, il est mort."

\section{/// Africando (Afrique de l'Ouest)}

"Africando est un concept, un label, que j'ai créé pour faire vivre la salsa des années 1960. En wolof, Africando veut dire 'les Africains ensemble'. En espagnol, ce serait plutôt "Allez I'Afrique". Ce terme qui sonne bien et qui a du sens correspond selon moi à toute la diaspora noire. "Africando est un défi que je me suis lancé en 1991 et que j'ai pu mener à bien grâce à la complicité de Boncana Maïga. Je l'ai rencontré en 1988, par l'intermédiaire de mon ami Constant à Abidjan, alors que je cherchais un producteur artistique et arrangeur pour Djina Mousso, l'album de Nahawa Doumbia. Nous sommes tombés d'accord, je lui ai remis l'argent pour la production et je suis parti. Après avoir entièrement terminé et mixé l'album, Boncana Maïga m'a apporté la bande et m'a rendu l'argent qu'il n'avait pas dépensé. C'est un homme de confiance et de talent. Ce premier album que nous avons fait ensemble a été un grand succès au Mali. "C'est en 1992, à Conakry, alors que je sortais le disque d'Oumou Dioubaté, que j'ai fait part à Boncana Maïga du projet que j'avais d'un groupe de salsa africaine, Africando. J'avais pensé réaliser le premier album à Paris avec trois chanteurs sénégalais, Pap Seck, Medoune Diallo et Nicolas Menheim.

Mais Boncana trouvait que Paris n'avait pas le son salsa. 'Si tu veux le vrai son salsa, il faut aller à Cuba ou à New York', m'a-t-il dit. Comme il a vécu une dizaine d'années à Cuba [il faisait partie des jeunes artistes musiciens envoyés à Cuba pour se former par l'État malien, dirigé de 1960 à 1968 par le tiers-mondiste Modibo Keïta], il n'avait pas envie d'y retourner, mais il était partant pour New York. On est parti là-bas à l'aventure et on a fait deux albums : des souvenirs inoubliables. “Depuis, Africando n'a cessé de s'ouvrir. Tous les artistes africains qui aiment la salsa et qui ont de bonnes chansons dans ce style peuvent rejoindre le groupe, qui est aujourd'hui reconnu par les amateurs de salsa du monde entier. Les Cubains d'Orquesta Aragon ont repris Yay Boy de l'album Sabador, qui est devenu un tube planétaire. Jean-Jacques Goldman nous a félicité pour la reprise d'Aïcha interprétée par Nicolas sur l'album Baloba! Carlos Santana a adoré le medley de ses chansons. Ce sont de belles satisfactions! » 